also zweifellos durch den Formaldehyd bezw. das Paraform eine Katalyse des Wasserstoffsuperoxyds bezw. der Metallsuperoxyde ein, undans cheinend wird durch das Alkalihydroxyd in statu nascendi das Paraform entpolymerisiert.

Ist die Reaktion an sich schon merkwürdig, so ist die explosionsartige Heftigkeit ganz besonders bemerkenswert, mit welcher sie verläuft, wenn man relativ geringe Mengen Wasser anwendet, und ist es dies Verhalten speziell, welches man zur Wohnungsdeșinfektion benutzen kann. Unter den von mir in Gemeinsohaft mit dem Bakteriologen, Herrn W e s e $\mathbf{n} b$ e $\mathbf{g}$, ausgearbeiteten Mischungsverhältnissen zwischen Paraform und Superoxyd wird beim Übergießen dieses „Autan"gemisches mit der gleichen Gewichtsmenge Wasser die Reaktion nach kurzer Zeit so heftig, daß nicht nur das Paraform vergast, sondern fast die Gesamtmenge Wasser, die den Forderungen F $\mathrm{l} \ddot{\mathrm{u}} \mathrm{g} g$ e $\mathrm{s}$ entsprechend das Vierfache des Formaldehyds beträgt, in Dampfform in die Luft gewirbelt wird und sich momentan im ganzen Raume verteilt. Nach den Versuchen von W es en berg genügt $1 \mathrm{~kg}$ dieser Paraformmischung mit 1 Liter Wasser nicht nur, um einen Raum von $30 \mathrm{cbm}$ Inhalt selbst bei Anwesenheit von sehr resistenten Bakterien (Staphylokokken) sicher zu desinfizieren, sondern auch eine solch andauernde Wasserübersättigung der'Luft herbeizuführen, daß das Hygrometer erst nach längerer Zeit unter den Sättigungspunkt geht. Da gerade dieses die Hauptbedingung eines völligen Erfolges ist, läßt sich Formaldehydlösung an Stelle des Paraforms deshalb nicht verwenden, weil man, abgesehen von der Oxydation eines großen Teiles des Formaldehyds vor Eintritt der Reaktion, durch dieselbe zu wenig Wasserdampf erbält, bezw. bei Zugabe der richtigen Wassermenge, also bei Verdünnung eines Liters Formalin auf vier Liter, überhaupt keine Verdampfung mehr eintritt. Abgesehen von dieser sicheren Erfüllung der F l ü g g e schen Bedingungen besitzt das Paraformgemisch noch den Vorteil, in fester Form anwendbar, leicht transportabel und in Form von Tabletten und Tafeln komprimierbar zu sein. In dieser Form hat es sich speziell zur Desodorierung von Räumen, und in Pulverform insbesondere auch zu solcher von Leichen bereits bewährt und dürfte in dieser Richtung noch vielfacher Anwendungsweise fähig sein, wie beispielsweise zur Desinfektion von Gegenständen (Stoffen, Kleidern, Briefen, Büchern), die man nicht benetzen will. Sein Hauptwert liegt aber auf dem Gebiete der Wohnungsdesinfektion, die es ermöglicht, ohne Anwendung von Feuer, ohne Benutzung eines Apparates eine Desinfektion von Wohn- und Krankenzimmern und auch von solchen Räumen vorzunehmen, in welchen die Anwendung F l ü g g e scher Apparate kaum möglich war, wie in Schränken, Fuhrwerken, Eisenbahncoupés oder Räumen mit leicht brennbarem Inhalt. Vor allem aber dürfte die neue Methode überall sich leicht Eingang verschaffen, wo Desinfektionsapparate, wie sie ja jetzt in jeder Großstadt eingeführt sind, nicht vorhanden sind, so in kleineren Städten, Dörfern, Landhäusern und einsamen Gehöften usw. Dort dürfte man nunmehr von der Anschaffung eines Desinfektionsapparates absehen können und die Autanmischung anwenden, zu deren Benutzung nichts gehört, als ein großer Eimer und eine Kanne Wasser, und welche noch vor allem den Vorzug hat, infolgeder intensiven, in wenigen Sek unden verlaufenden Verd a m p f ng, den Formaldehyd in soleher Konzentration im $R$ a u $m$ e zu verteilen, daß eine spätere Verdünnung der Gase durch etwaige Undichtigkeiten nicht mehr in Frage kommt, und man vom Verkleben und Verstopfen der Türritzen, Fensterritzen, Schlüssellöcher usw. absehen kann. Da außerdem die Verwendung einer Ammoniakbombe in den meisten Fällen unnötig sein dürfte, weil beim Einbringen von Salmiak in den alkalischen Rückstand genügende Mengen Ammoniak frei werden, um den größten Teil des Formaldehydes zu binden, dürfte die Autanmethode das denkbar einfachste Formaldehyddesinfektionsverfahren darstellen.

\section{Über die Addition von Alkali an Indigo.}

(Siebente Mitteilung über Indigofärberei.) Von A. Binz.

(Eingeg. d. 15./6. 1906.)

Neutrales, indigodisulfosaures Natrium ist, wie A. W a lter und ich gezeigt haben ${ }^{1}$ ), imstande, Nalrium- und Calciumhydroxyd zu addieren. Hieraus und aus anderen Tatsachen, die sich beim Studium der Indigoreduktion ergeben hatten ${ }^{2}$ ), insbesondere der Reduzierbarkeit von Indigo durch eine Zinkanode, schlossen wir auf folgenden Reaktionsverlauf in der Küpe :

Zunächst bilden sich um die Farbstoffpartikelchen Schichten von Natronindigo oder Kalkindigo; diese verlieren bei der Reduktion Sauerstoff, gehen als Indigweißsàlze in Lösung und werden stetig wieder erneut und reduziert, bis die Gesamtmenge des Farbstoffes umgesetzt ist. Demgemäß wären z. B. die Reaktionen in der Hydrosulfitküpe folgende :

1) $\mathrm{C}_{16} \mathrm{H}_{10} \mathrm{~N}_{2} \mathrm{O}_{2}+2 \mathrm{NaOH}=\mathrm{C}_{16} \mathrm{H}_{10} \mathrm{~N}_{2} \mathrm{O}_{2} \cdot 2 \mathrm{NaOH}$

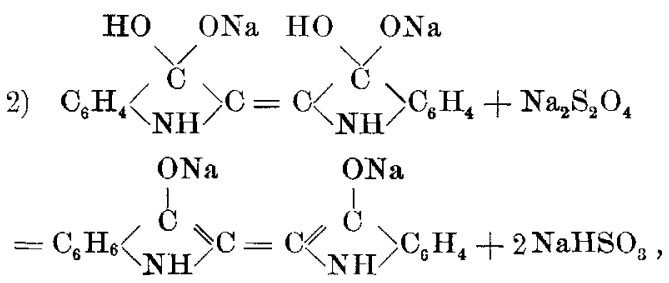

was sich leicht auch auf die Zinkstaubküpe und die Ferrosulfatküpe übertragen läßt.

Die erste dieser Gleichungen ist nun durch ein in der Folge angemeldetes Patent $^{3}$ ) der $\mathrm{Bad} \mathrm{i}$. s chen Anilin - und Soda-Fabrik in erfreulicher Weise gestützt worden; in ihm werden

1) A. B i n z und A. W a I t e r, Chem. Industr. 26, 248 (1903); Z. f. Farb.- u. Textilind. 2, 435 (1903); diese $Z$. 17, 40 (1904).

2) A. B i n z, Z. f. Elektrochem. 5, 103 (1898).

A. B in $z$ und F. R u n g, diese $Z$. 13, 428 (1900).

3) D. R. P. 158625 vom 26./10. 1903 . 
Additionsprodukte aus Alkali resp. Erdalkali und Indigo beschrieben, deren Existenz $W$ a 1 t e $r$ und ich vorausgesagt haben. Die Reindarstellung dieser Körper bildet erhebliche Schwierigkeiten. Immerhin hat sich analytisches Material gewinnen lassen, welches in die Additionsreaktionen des Indigos und ihre Rolle in der Küpe weiteren Einblick gewährt.

\section{Addition von Natriumbydroxyd an In digo.}

Versuch 1. In Anlehnung an eine der Methoden der Patentbeschreibungen wurden $20 \mathrm{~g}$ 99\%iges Indigopulver der Badischen Anilin- und Soda-Fabrik in $840 \mathrm{ccm}$ filtrierter alkoholischer Natronlauge, dargestellt aus $96 \mathrm{~g}$ Natriumhydroxyd, $104 \mathrm{com}$ Wasser und $640 \mathrm{cem}$ Alkohol, 11 Tage lang geschüttelt. Darauf wurde ein Teil abgesaugt. Der Rückstand erschien nach gründlichem Abpressen auf Ton als trockenes, dunkelgrünes Pulvef, das sich nicht alkalisch anfühlte. In Wasser, ebenso bei längerem Liegen an der Luft, wurde es blau; langsamer als durch Wasser wird die Substanz durch Alkohol in Indigo und Alkali gespalten.

Zur Analyse wurde ein Teil des auf Ton abgepreßten in Wasser suspendiert und mit verdünnter Schwefelsäure in geringem Utberschu $B$ versetzt. Auf einem tarierten Filter hinterblieben $1,489 \mathrm{~g}$ Indigo, Filtrat und Waschwasser ergaben nach dem Eindampfen und Glühen mit etwas Ammoniumcarbonat 0,7608 g Natriumsulfat, entsprechend 1,90 Mol. Alkali auf $1 \mathrm{Mol}$. Indigo.

Versuch 2. Der Rest des Reaktionsgemisches wurde weitere 8 Tage geschüttelt. Bei der Analyse fanden sich 2,106 $\mathrm{g}$ Indigo und $1,146 \mathrm{~g}$ Natriumsulfat, entsprechend $2 \mathrm{Mol}$. Alkali auf $1 \mathrm{Mol}$. Indigo.

Versuch 3. Da sich der Natronindigo nicht ohne Zersetzung mit Alkohol auswaschen läßt, so ergibt sich aus diesen Zahlen nicht, wieviel des Alkalis chemisch gebunden war, und wieviel trotz Absaugens und Abpressens mechanisch adhärierte.

Um darüber einen Anhalt zu bekommen, wurde Indigo in demselben Verhältnis wie vorher gründlich mit alkoholisehem Natron gemischt und dann sofort abgesaugt und abgepreßt. Grünfärbung trat nicht ein. Die Hydrolyse und Analyse einer Probe ergab $0,6797 \mathrm{~g}$ Farbstoff und $0,445 \mathrm{~g}$ Natriumsulfat, entsprechend 0,890 Mol. Natriumhydroxyd auf 1 Mol. Indigo.

Es läßt sich nicht entscheiden, ob in diesem Falle die Gesamtmenge des Alkalis mechanisch anhaftete, oder ob doch ein Teil chemisch gebunden war. Ich bin geneigt, letzteres anzunehmen, da die Addition von Alkali an Indigkarmin sofort erfolgt, die Neigung der Carbonylbindungen, sich aufzurichten, also sehr groß ist. Aber selbst wenn man bei Versuch 3 das Alkali als mechanisch anhaftend betrachtet, und demnach $0,89 \mathrm{Mol}$. von den Zahlen der Versuche 1 und 2 abzieht, so ergeben sich dennoch als chemisch gebunden:

$$
\begin{aligned}
& \text { Ber. für Gefunden } \\
& \mathrm{C}_{12} \mathrm{H}_{10} \mathrm{~N}_{2} \mathrm{O}_{2} \cdot \mathrm{NaOH} \quad \text { 1. } 2 \text {. } \\
& \text { Moleküle } \\
& \mathrm{NaOH} \quad 1,00 \quad 1,01 \quad 1,11
\end{aligned}
$$

Demnach scheint es, daß vorzugsweise die eine Hälfte des Indigomoleküls Alkali festhält. Das entspricht dem Verhalten des Farbstoffes gegen
Säuren : Das Disulfat $\mathrm{C}_{16} \mathrm{H}_{10} \mathrm{~N}_{2} \mathrm{O}_{2} \cdot 2 \mathrm{H}_{2} \mathrm{SO}_{4}^{4}$ ) ist unbeständig und nicht isolierbar, dagegen sind die Salze

$$
\begin{gathered}
\mathrm{C}_{16} \mathrm{H}_{10} \mathrm{~N}_{2} \mathrm{O}_{2} \cdot \mathrm{H}_{2} \mathrm{SO}_{4}, \\
\mathrm{C}_{16} \mathrm{H}_{10} \mathrm{~N}_{2} \mathrm{O}_{2} \cdot \mathrm{HCl} \text { und }\left(\mathrm{C}_{16} \mathrm{H}_{10} \mathrm{~N}_{2} \mathrm{O}_{2}\right)_{2} \mathrm{P} t \mathrm{Cl}_{6} \mathrm{H}_{2}
\end{gathered}
$$

beständige kristallinische Körper ${ }^{5}$ ).

Das bei Versuchen 1, 2 und 3 hydrolytisch regenerierte Indigotin erwies sich bei der Titration mit Permanganat ${ }^{6}$ ) als genau so rein wie das Ausgangsmaterial.

Das beim Versuch I vom Rückstand abgesaugte zeigte die rotbraune Farbe, welche Alkoholatlösungen mit der Zeit annehmen. Auf Filtrierpapier entstand keine Spur einer Küpenfärbung. Reduktion war also durch das Schütteln des Indigos mit alkoholischer Natronlauge nicht eingetreten. Dagegen hatte sich etwas Natronindigo gelöst. Mit Salzsäure fielen aus $84 \mathrm{ccm} 0,1137 \mathrm{~g}$ Indigo aus. Die Titration ergab $58,1 \%$ Indigotin.

Rascher als durch Schütteln läßt sich Indigo durch Erwärmen mit konz. wässeriger oder mit alkoholischer Natronlauge mit Alkali resp. Alkoholat vereinigen. Die Reaktion beginnt bereits bei $40^{\circ}$ und ist bei $60^{\circ}$ in wenigen Minuten beendet, wie an der charakteristischen Grünfärbung des Indigos ersichtlich ist.

\section{Addition von Phenolnatrium an Indi go.}

Versuch 1. $59 \mathrm{~g}$ reines Phenol wurden zusammen mit der für Phenolnatrium berechneten Menge Natrium $(14,4 \mathrm{~g})$ in absolutem Alkohol zu $150 \mathrm{ccm}$ aufgelöst. Mit $20 \mathrm{ccm}$ dieser Lösung wurden $2 \mathrm{~g}$ Indigopulver erwärmt. Während bei dem entsprechenden Versuch mit alkoholischer Natronlauge schon bei etwa $40^{\circ}$ Grünfärbung sichtbar wird, verändert der Farbstoff in diesem Falle erst bei $60^{\circ}$ seine Nuance.

Es wurde 20 Minuten unter Rühren bis auf $77^{\circ}$ erwärmt. Beim Absaugen und Abpressen auf Ton hinterblieb eine schwarzgrüne Masse. Ein Teil wurde hydrolysiert und filtriert. Rückstand : 1,147 g Indigo.

Das Filtrat samt Waschwasser betrug $150 \mathrm{ccm}$. Die Titration des Phenols nach Ko p pe s c h a a r durch Bromierung mit Bromnatrium und Natrium. bromat in bekanntem ÜberschuB, Zusatz von Jodkalium und Rücktitration mit Thiosulfat ergab 0,4918 g Phenol, entsprechend 1,195 Mol. des letzteren auf 1 Mol. Indigo.

Versuch 2. Dasselbe Reaktionsgemisch wie beim vorigen Versuch wurde eine Stunde lang bei $80^{\circ}$ erwärmt. Die Analyse ergab 0,7752 $\mathrm{g}$ Indigo auf $0,2958 \mathrm{~g}$ Phenol, entsprechend 1,06 Mol. des letzteren auf $1 \mathrm{Mol}$. Farbstoff.

$\mathrm{Da}$ trotz der dreimal längeren Reaktionsdauer nicht mehr Phenolnatrium addiert worden war, wie bei Versuch 1 , so ist anzunehmen, daß in beiden Fällen die Reaktion ihr Ende erreicht hatte.

4) D. R. P. 121450 , B a dis ohe An ilin und S od a-F a b r i k 1901.

5) A. B inz und A. K u f f e r at h, Liebigs Ann. 325, 196 (1902).

6) Nach Vorschrift im Indigobuch der Badischen Anilin- und Soda-Fabrik S. 18 mit 0,01\%iger Indigosulfosäurelösung ausgeführt. 
Versuch 3. Zur Bestimmung des mechanisch adhärierenden Phenolnatriums wurde ein Reaktionsgemisch ohne Erwärmen angesetzt und dann sofort abgesaugt und auf Ton abgepreßt. Es fanden sich 1,062 g Indigo und 0,1751 g Phenol, entsprechend 0,46 Mol. Phenolnatrium auf $1 \mathrm{Mol}$. Farbstoff. Zieht man diese Menge von den bei 1 und 2 gefundenen Phenolmengen $a b$, so ergeben sich als chemisch gebundenes Phenolnatrium :

$$
\begin{array}{ccc}
\text { berechnet für } & \multicolumn{2}{c}{\text { gefunden }} \\
\begin{array}{c}
\mathrm{C}_{16} \mathrm{H}_{10} \mathrm{~N}_{2} \mathrm{O}_{2} \cdot \mathrm{C}_{6} \mathrm{H}_{5} \mathrm{ONa} \\
\text { Moleküle }
\end{array} & 1 . & 2 . \\
\text { Phenolnatrium 1,00 } & 0,73 & 0,60
\end{array}
$$

Die Verwandtschaft des Indigos zum Phenolnatrium ist also schwächer wie die zum alkoholischen Natrium, was sich schon darin äußert, daß ersteres zu seiner Einwirkung einer höheren Tempe. ratur bedarf, als letzteres. Indessen zeigt schon die charakteristische Grünfärbung beim Erwärmen von Indigo mit Phenolnatrium an, daß wirklich eine Reaktion stattfindet, und daß Indigo, ähnlich wie Chinon ${ }^{7}$ ), Phenolnatrium addiert.

\section{Beschleunigung der Indigo- reduktion dureh Alkohol.}

Wenn wirklich Gleichung 2 die zweite Phase der Verküpung darstellt, dann muß letztere durch Alkohol beschleunigt werden, da dieser die Hydrolyse zurückdrängt und somit die Addition von Alkali an Indigo erleichtert, also diejenige Verbindung entstehen läßt, welche das für die Reduktion notwendige Zwischenprodukt ist.

Zur Prüfung dieses Schlusses wurden bei einer Reihe von Versuchen je 2 g Indigopulver B. A. $u$. S. F., $1 \mathrm{~g}$ Zinkstaub (enthaltend 75,22\% met. Zink) und $3 \mathrm{~g}$ Natriumhydroxyd in großen Reagensgläsern miteinander in Reaktion gesetzt, und zwar einerseits in wässeriger, andererseits in alkoholischer Lösung.

$\mathrm{Zu}$ diesem Zwecke wurde der Indigo mit $0,25 \mathrm{ccm}$ Alkohol befeuchtet und dann vollends mit der Lauge angeteigt. Der geringe anfängliche Alkoholzusatz kommt für die Verküpung nicht in Betracht, ist aber zur Erzielung einer gleichmäßigen Paste notwendig, da Indigo sich mit Wasser allein nur schwer netzt. Während der Verküpung wurde langsam umgerührt, nach einer bestimmten Zeit fand Zusatz von Wasser bis zu einem Gesamtvolumen von $80 \mathrm{ccm}$ statt, worauf das Gefäß verschlossen und zur gründlichen Durchmischung des Inhaltes einige Male umgekehrt wurde. Dann wurde die Küpe in ein graduiertes Gefäß filtriert; die zuletzt durchgelaufenen $10 \mathrm{ccm}$ ergaben durch $0 \mathrm{xy}$ dieren mit Luft, Filtrieren durch ein tariertes Filter, Ansäuern, Auswaschen und Trocknen bei $110^{\circ}$ die Menge des reduzierten Indigos, und damit ein $\mathrm{Maß}$ für die Geschwindigkeit der Verküpung.

In allen Vergleichsfällen waren die Versuchsbedingungen auch in allen selbstverständlichen und

\begin{tabular}{|c|c|c|c|c|c|}
\hline & $\begin{array}{l}\text { wăsse- } \\
\text { riger } \\
\text { Küpe }\end{array}$ & $\begin{array}{l}\text { Temp. } \\
\text { Grad }\end{array}$ & $\begin{array}{c}\text { alkohol. } \\
\text { Kupe }\end{array}$ & $\begin{array}{l}\text { Temp. } \\
\text { Grad }\end{array}$ & 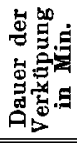 \\
\hline 1. & 0,0227 & $18-28$ & 0,1572 & $18-38$ & 8 \\
\hline 2 . & 0,0118 & $18-28$ & 0,2138 & $18-38$ & 10 \\
\hline 3. & - & - & 0,1741 & 18 & 10 \\
\hline 4. & 0,0026 & 18 & 0,0702 & 18 & 10 \\
\hline 5. & 0,0006 & 18 & 0,1104 & 18 & 10 \\
\hline 6. & 0,0038 & 18 & 0,0851 & 18 & 10 \\
\hline 7. & 0,0149 & 50 & 0,2079 & 50 & 10 \\
\hline 8. & 0,0108 & 50 & 0,2234 & 50 & 10 \\
\hline
\end{tabular}
darum hier nicht erwähnten Einzelheiten genau dieselben.

7) J a cks on und O ens la ger, Berl. Berichte 28, 1616 (1895).
Die Resultate waren folgende:

g Indigo aus je $10 \mathrm{com}$ Filtrat von:

Die Anfangstemperaturen waren bei 1.-6. stets etwa $18^{\circ}$, auch wurde die alkoholische Lauge, die sich bei der Darstellung erwärmt, stets gekühlt angewendet. Daß trotzdem die Temperatur bei den ersten Küpen auf $28 \mathrm{bzw} .38^{\circ}$ stiegen, rührt von den Reaktionswärmen her. Thre Verschiedenheit beeinträchtigt den Vergleich zwischen alkoholischen und wässerigen Küpen nicht, denn die Ausbeute bei Küpe 3, welche von Anfang an gekühlt wurde, steht zwischen der der alkoholischen Küpen 1 und 2.

Überblickt man nun das Gesamtergebnis, so zeigt sich, $d$ a $B$ die Verküpung in den $a_{1} 1 \mathrm{koholisehen} \mathrm{Küpen} \mathrm{etwa} 30 \mathrm{mal}$ rascher verläuft, als in den wässe. ri g e n. Allerdings kommen auch in den beiden Vertikalreihen bei gleichen Versuchsbedingungen Unterschiede vor. Sie erklären sich daraus, daß es kaum möglich ist, Zinkstaub gleichmäßig mit Indigo in Reaktion zu bringen und die Küpen ohne wechselnde Verluste zu filtrieren. Diese Unterschiede sind aber verschwindend klein gegenüber denen in den Horizontalreihen.

Reaktionsgleichung 2 findet darin eine, wie mir scheint, starke Stütze.

Man könnte einwenden, alkoholisches Natron reduziere Indigo, und darum befördere Alkohol die Verküpung. Dieser Einwurf trifft nicht zu, denn Indigo wird durch alkoholisches Natron nicht im geringsten verküpt. Nur in der Siedehitze findet eine geringfügige Einwirkung statt, dieselbe führt aber nicht zu Indigweißnatrium, sondern zu einem Zerstörungsprodukt, das sich in Alkali grün, in Säuren und in Ather rot löst8).

Da Alkohol lösend auf Natronindigo wirkt, wie oben gezeigt wurde, so befördert er jedenfalls auch dadurch die Verküpung. Auch bei der gewöhnlichen Küpe dürften kontinuierliche kleine Mengen Natron oder Kalkindigo in Lösung gehen und in dieser Form reduziert werden.

Diese von mir schon früher ausgesprochene Vermutung ${ }^{2}$ ) gewinnt durch folgende Versuche an Wahrscheinlichkeit :

$2 \mathrm{~g}$ Indigo wurden mit $1 \mathrm{~g}$ Zinkstaub und einigen Tropfen Alkohol angeteigt und dann mit $15 \mathrm{ccm} 49 \%$ iger Natronlauge 2,5 Minuten verrührt. Nach dem Auffüllen auf $87 \mathrm{ccm}$ Wasser, Umschütteln und Filtrieren zeigte sich keine Spur von Küpe.

8) Vgl. A. B in z und F. R u n g, diese Z. 12, 519, Anm. 35 (1899).

9) Z. f. Elektrochem. 9, 599 (1903). 
Dasselbe Resultat ergab ein zweiter Versuch, bei dem die Lauge mit 1 Raumteil Wasser verdünnt wurde. Erst beim Verdünnen von $5 \mathrm{ccm}$ der $\mathrm{Na}$ tronlauge mit $10 \mathrm{ccm}$ Wasser und sonst gleichen Versuchsbedingungen wurde schwache Küpenbildung beobachtet. Setzt man also die Löslichkeit des Natronindigos durch Konzentration des Alkalis herab, so kommt die Küpenbildung zum Stillstand.

H. B u cherer10) hat mit Recht bemerkt, die Erforschung der inneren Vorgänge bei der Verküpung des Indigos sei von der allgemeinsten Bedeutung zur Aufklärung auch anderer Reduktionsprozesse. Vielleicht kann man demnach den Unterschied zwischen alkalischen und sauren Reduktionen darin erblicken, daß bei ersteren Anlagerungen von Alkali und Sauerstoffwegnahme, bei letzteren Anlagerung von Wasserstoff stattfindet.

\section{Optische Analyse der Industriegase.} Von F. HABER. ${ }^{1}$ )
(Eingeg. d. 11./6. 1906.)

Meine Herren! In jedem Zweige der Technik besteht ein lebhaftes Bedürfnis nach automatisch wirkenden Vorrichtungen, welche die Augenblickswerte veränderlicher Betriebsgrößen anzeigen oder registrieren. Dieses Bedürfnis. hat die Sohaltbrettinstrumente in der Elektrotechnik, die Wasserstandszeiger und Manometer im Kesselbetrieb, die Gasubren in der Leuchtgasindustrie, die Thermo- und Pyrometer im Heizwesen zu unentbehrlichen Werkzeugen gemacht. Gleichartige Werkzeuge für die fortlaufende Kontrolle der chemischen Zusammensetzung besitzen wir im allgemeinen nicht. Die chemischen Veränderungen der Stoffe entziehen sich meist der Bestimmung durch so einfache Mittel, wie sie für die Anzeige von Spannungen, Stromstärken, Drucken, Geschwindigkeiten, Temperaturen und Höhenunterschieden zu Gebote stehen. Die einfachsten chemischen Stoffe aber, die Gase, erlauben immerhin, in einer Reihe von Fällen die Leistung des analytischen Chemikers einem selbsttätigen Apparat zu übertragen, so daß die Tätigkeit des Beobachters auf die Ablesung einer Skala sich beschränkt, auf welcher der Apparat das Analysenergebnis anzeigt oder registriert. Man hat insbesondere für die Feuerungstechnik eine Reihe solcher Konstruktionen ausgearbeitet. Der Nutzeffekt einer Feuerung hängt in empfindlicher Weise vom Luftüberschuß ab. Die ideale Fouerung würde bei vollkommener Verbrennung das theoretische Rauchgas liefern, welches keinen freien Sauerstoff mehr enthält und seiner Zusammensetzung nach aus der Analyse des Brennstoffes und der stets gleich bleibenden Zusammensetzung der Luft zu berechnen ist. Das praktische Rauchgas ist ein Gemisch aus theoretischem Rauchgas und überschüssiger Luft. Das Mischungsverhältnis geht diesem Zusammenhang gemäß aus

10) Z. f. Farb.- u. Textilind. 3, 25 (1904).

1) Vortrag, gehalten auf der Hauptversammlung des Vereins deutscher Chemiker zu Nürnberg, am 8./6. 1906. einer einzigen analytischen Bestimmung, sei es aus der eines Einzelbestandteiles, wie Kohlensäure oder Sauerstoff, sei es aus der einer Gesamteigenschaft, wie der des spezifischen Gewichts, hervor. In vielen anderen Fällen liegen die Dinge insofern ähnlich, als eine Einzelbestimmung imstande ist, die für die Beurteilung der Betriebsverhältnisse erforderliche Kenntnis der Zusammensetzung zu vermitteln. Wohl erlaubt eine solche Einzelbestimmung natürlich nicht, die Prozentwerte der Bestandteile eines komplizierten Gasgemenges zu erkennen. Aber sehr häufig bedingt die Herkunft der Gase, daß bestimmten Änderungen des gasliefernden Prozesses auch bestimmte Änderungen der Zusammensetzung entsprechen, so daß man durch die fortlaufende Kontrolle einer Eigenschaft des Gasgemisches den Gang des ganzen Prozesses kontrolliert.

Es ist mir nun gelegentlich aufgefallen, daB unter den Bestimmungen einer Gesamteigenschaft diejenige der optischen Dichte, $d . h$. der Lichtbrechung, einige Vorzüge besitzt. Sie ermöglicht Ausführungsformen, bei denen weder Sperrflüssigkeiten, noch bewegte Teile für die Wirksamkeit des Apparates erforderlich sind. Dadurch gewinnt diese Bestimmungsweise an allgemeiner Anwendbarkeit, und die Gefahr unvermerkt eintretender Fehler, die von der Abnutzung der bewegten Teile herstammen, fällt weg.

Das die Lichtbrechung der Gase mit den einfachen Hilfsmitteln eines Gasprismas und Fernrohrs genau bestimmbar ist, hat schon $\mathrm{Dulon} \mathrm{g}^{\mathrm{I}}$ ) vor 80 Jahren erwiesen. Daß die Lichtbrechung eines Gasgemisches auf sehr einfache Weise aus der Licht. brechung der Einzelbestandteile und ihrem Partial. druck berechenbar ist, weiß man sogar noch etwas länger. Bezeichnet man nämlich den um eins verminderten Brechungsexponenten als brechende Kraft, so addieren sich bei einem Gasgemische nach $\mathrm{Biot}$ und A ra g o ${ }^{2}$ ) die brechenden Kräfte in derselben Weise zur brechenden Gesamtkraft, wie die Partialdrucke zum Gesamtdruck ${ }^{3}$ ).

Man hat eine Benutzung dieser Tatsachen für die Zwecke der technischen Gasanalyse bisher, so. viel mir bekannt ist, nicht versucht. Das mag zum Teil daran liegen, daß der Arbeitskreis der tech. nischen Gasanalyse und der optisehen Physik kaum Berührung haben. Zum anderen Teil dürfte aber ein Vorurteil dem Versuche im Wege gestanden haben. Die Brechungskoeffizienten der reinen Gase unterscheiden sich meist erst in der vierten Dezimale. Die Untersohiede, welche die technische Gas.

1) Ann. de chim. et de phys. 31, 154 (1826); Poggendorfs Ann. 6, 393 (1826).

2) Mém. de l'Acad. de France \%, 301 (1806); Gilbert Ann. 25, 345 (1807); 26, 36 (1807).

3) Das Gesetz von B i o t und A r a go ist ein Grenzgesetz ebenso wie das Dalton sche, nach welchem die Summe der Partialdrucke gleich dem Gesamtdruck eines Gasgemenges ist. Man vgl. die Untersuchungen, welche $R$ a $\mathrm{m}$ s a y und Tra. vers (Proc. of the Royal Soc. 62, 225 [1897]); E. H. J. Cu n a e u s (Z. physikal. Chem. 36, 232 [1902]) und G. C. Gerrits (Dissertation Amsterdam 1901) nach dem Interferenzverfahren von Lord Rayleigh (Proc, of the Royal Soc. of London 59, 203 [1896]) ausgeführt haben. 